\title{
Mesoscale modeling of dynamic recrystallization behavior, grain size evolution, dislocation density, processing map characteristic, and room temperature strength of Ti-6Al-4V alloy forged in the $(\alpha+\beta)$ region
}

\author{
Hiroaki Matsumoto ${ }^{\mathrm{a}}{ }^{*}$, Vincent Velay ${ }^{\mathrm{b}}$ \\ a Department of Advanced Materials Science, Faculty of Engineering, Kagawa University, 2217-20 Hayashi-cho, Takamatsu, Kagawa 761-0396, Japan \\ ${ }^{\mathrm{b}}$ Université de Toulouse, CNRS Mines Albi, INSA, ISAE, ICA (Institut Clément Ader), Campus Jarlard, 81013 ALBI Cedex 09, France
}

\begin{abstract}
A B S T R A C T
This work focuses on a series of microstructural predictions regarding the dynamic recrystallization (DRX) behavior, change in grain size and dislocation density. Additionally, this study includes the prediction of the room-temperature-strength and the processing map characterization of Ti-6Al-4V alloy after forging in the $(\alpha+\beta)$ region. The experimental tests of the forgings reveal that the mechanism of microstructural conversion depends on the testing conditions. That is, superplastic behavior is exhibited at lower strain rates and higher temperatures. With an increasing strain rate and decreasing temperature, DRX occurs in a continuous manner. A set of constitutive equations that model the microstructural evolution, processing map characteristics and room temperature strengths is established by optimization of experimental data, followed by implementation into the DEFORM-3D software package. There is satisfactory agreement between the experimental and simulated results. Thus, a series of constitutive models (established in this work) is found to enable the reliable prediction of properties of Ti-6Al-4V alloy after forging in the $(\alpha+\beta)$ region.
\end{abstract}

Keywords:

Ti-6Al-4V alloy

Dynamic recrystallization

Microstructural prediction

Processing map

Finite element simulation

\section{Introduction}

During hot deformation of metallic alloys, dynamic recovery (DRV) and dynamic recrystallization (DRX) occur thereby resulting in microstructural conversion [1]. The types of DRX process include (1) discontinuous dynamic recrystallization (DDRX), in which new grains are formed by heterogeneous nucleation and growth processes, and (2) continuous dynamic recrystallization (CDRX), in which subgrains with low-angle boundaries are formed that subsequently evolve into grains with a high fraction of high-angle boundaries, with increasing plastic strain.

Over the past few decades, phenomenological microstructureevolution models based on the Johnson-Mehl-AvramiKolmogorov (JMAK) formulation [2] have been developed. These models succeeded in microstructural prediction during hotworking [3-7]. Additionally, the Avrami approach often provides

\footnotetext{
* Corresponding author

E-mail address: matsu_h@eng.kagawa-u.ac.jp (H. Matsumoto).
}

reasonable results. However, these models are usually only applied to specific or relatively narrow ranges with respect to the initial microstructure conditions, testing temperatures, strain rate, and strain. In addition, the physics-based models, such as the cellularautomaton (CA) model, the Monte-Carlo technique, and phasefield simulation have been developed over several years. These models make it possible for developing numerical solutions of the complex problems presented by microstructural evolution during thermomechanical processing within a unified frame. Despite their strengths, there are some drawbacks in that these models require excessive computer power to formulate a solution and the modeling is only applicable to selected local regions [8].

The Ti-6Al-4V alloy has been widely used for aerospace applications due to its excellent properties including high specific strength, fracture toughness and corrosion resistance. With respect to numerical modeling for microstructural prediction under hot working of Ti alloys, a considerable number of studies have been conducted [9-16] since in the 2000s. Among these reports, Ding et al. [9] and Wu et al. [10] simulated the microstructures on the basis of CA model. Furthermore, there are reports that the 
mesoscale and microscale internal state variables model can be applied to microstructural prediction [11-13]. Sun et al. succeeded in microstructural prediction for the TA15 alloy based on the developed dislocation-density rate equation [11]. In addition, DRX fraction and grain size have been also successfully predicted according to the JMAK model [14-16]. With respect to evaluation of hot forging property, processing map technique $[17,18]$ based on dynamic materials model (DMM) has been widely utilized for optimization of the process conditions.

The present work focuses on a series of microstructural predictions (i.e., DRX behavior, the change in grain size and dislocation density) and processing map characteristics for forgings of Ti-6Al$4 \mathrm{~V}$ alloy in combination with finite-element-method (FEM) analysis. In addition, the $0.2 \%$ proof strength (at room temperature) after forging is also estimated on the basis of the simulated microstructural factors and classical strengthening factors according to the Hall-Petch relation, the Bailey-Hirsch relation, and simple constituent-phase-mixture-rule. This work first experimentally identifies the forging property and microstructural evolution of the Ti-6Al-4V alloy with the equiaxed $(\alpha+\beta)$ starting microstructure. Next, constitutive formulas for predicting the microstructural evolution are established based on the optimization process from the experimental results, followed by implementation into FEM software (DEFORM-3D, v.10.2).

\section{Experimental procedures}

\subsection{Isothermal forging test}

The Ti-6Al-4V alloy (hereafter designated as Ti-64 alloy) in this work was a commercial-grade alloy with chemical compositions (in wt.\%) of $6.25 \mathrm{Al}, 4.30 \mathrm{~V}, 0.20 \mathrm{O}, 0.007 \mathrm{~N}$, and the balance comprised of Ti. The starting microstructure (as shown in Fig. 1) before isothermal forging is an equiaxed $(\alpha+\beta)$ microstructure having an average grain size of $2.25 \mu \mathrm{m}$ for $\alpha$ phase. The $\beta$ fraction for the initial condition is $7 \%$. Isothermal forging was performed using the computer-aided ThermecMastor- $Z$ equipment. During forging, process temperatures were $700{ }^{\circ} \mathrm{C}, 800{ }^{\circ} \mathrm{C}$, and $900{ }^{\circ} \mathrm{C}$, while the strain rates range from $10^{-3} \mathrm{~s}^{-1}$ to $10 \mathrm{~s}^{-1}$. Samples for forging were prepared by electron discharge machining to dimensions with a diameter of $8 \mathrm{~mm}$ and a height of $12 \mathrm{~mm}$. Induction heating of a specimen was carried out at $10^{\circ} \mathrm{C} / \mathrm{s}$ up to the testing temperature and maintained for $60 \mathrm{~s}$ to ensure the forging had a uniform temperature. The heat-up process was followed by forging at constant strain rate and to a height true-strain of approximately 0.7 . After a compression test, the obtained stress-strain curves were corrected by a friction correction (based on reports by Li et al. [19,20]) and

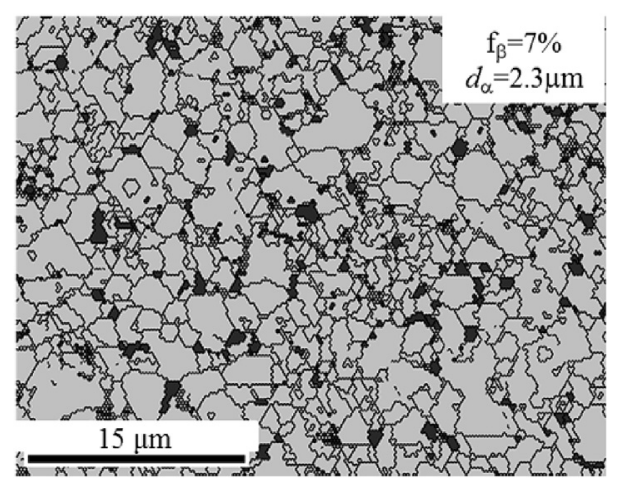

Fig. 1. Initial microstructure of equiaxed $(\alpha+\beta)$ microstructure for hot compression test shown by EBSD-phase map image (gray phase: $\alpha$ phase and black phase: $\beta$ phase). adiabatic correction (based on the report by Mataya and Sackschewsky [21]) in order to obtain accurate stress-strain curves.

\subsection{Microstructural analysis}

Microstructures were identified by conducting an electron backscatter diffraction (EBSD) analysis. In EBSD analysis, grain orientations were measured at step sizes of $0.12 \mu \mathrm{m}$ and $0.20 \mu \mathrm{m}$, and the EBSD data were post-processed by means of the TSL orientation imaging microscopy (OIM) data analysis software. The grain sizes of both high-angle-grains and low-angle-grains were measured using this software. In addition, the fraction of the DRX grain was identified by counting the equiaxed-fine-grain in which the DRX occurred.

\subsection{Predictions of microstructures and forging properties combined with FEM analysis}

Forging properties of Ti-64 alloy with the equiaxed $(\alpha+\beta)$ microstructure are analyzed by FEM utilizing the DEFORM-3D software package (v. 10.2) through a user-defined subroutine. The forging condition in the simulation is similar to the experimental condition. In FEM simulation, the friction coefficient between the die-tools and the workpiece was set as 0.7 . Due to the symmetry of the workpiece (with dimension of $8 \mathrm{~mm}$ in diameter and $12 \mathrm{~mm}$ in height), a quarter of it was taken as the object and the constraint was applied in the plane of symmetry. The data from the stressstrain curves obtained in this work, after the corrections were implemented, was programed into the FEM code.

The constitutive equations for modeling of microstructure and mechanical properties are summarized in Section 4.

\section{Results and discussion}

3.1. Experimental results of forging properties of Ti-64 with the equiaxed $(\alpha+\beta)$ starting microstructure

\subsubsection{Stress-strain curves}

Fig. 2 shows the stress-strain curves (tested at $700{ }^{\circ} \mathrm{C}, 800{ }^{\circ} \mathrm{C}$, and $900^{\circ} \mathrm{C}$ and the strain rates of (a) $10^{-3} \mathrm{~s}^{-1}, 10^{-2} \mathrm{~s}^{-1}, 10^{-1} \mathrm{~s}^{-1}$ and (b) $1 \mathrm{~s}^{-1}, 10 \mathrm{~s}^{-1}$ ) after corrections for friction and increasing temperatures. Extensive flow softening is observed with an increasing strain rate and decreasing testing temperature. For the testing conditions at higher temperature and lower strain rates, a steady stress state is exhibited. This steady state case implies that the DRV or CDRX is the dominant mode for microstructural conversion. Additionally, the continuous flow softening seen at lower temperatures and higher strain rates may be attributed to flow instability, as already reported in research regarding hot deformation behavior of Ti alloys [22].

Fig. 3 shows the relationship between the stress value at a true strain of 0.1 and the strain rate in Log-Log scale. The slope of $m$ given by $\delta \sigma / \delta \dot{\varepsilon}$ from Fig. 3 corresponds to strain-rate sensitivity. The $m$ values are also illustrated in Fig. 3, revealing the linear relationships at testing temperatures of $700^{\circ} \mathrm{C}$. The $m$ value is seen to be higher with an increasing temperature. At testing temperatures of $800{ }^{\circ} \mathrm{C}$ and $900{ }^{\circ} \mathrm{C}$, there are two slopes at the low strain rate region and high strain rate region. The $m$ value at $900{ }^{\circ} \mathrm{C}$ and the lower strain rate region is noted to exceed 0.3, indicating the occurrence of superplastic behavior.

The apparent activation energy $Q$ for hot deformation is determined by assuming the strain rate follows an Arrhenius type equation as follows: 
(a)

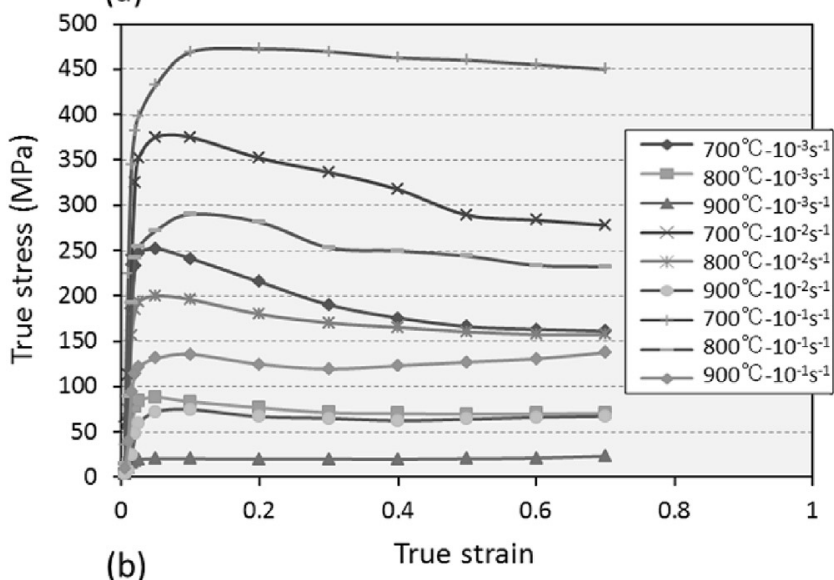

(b)

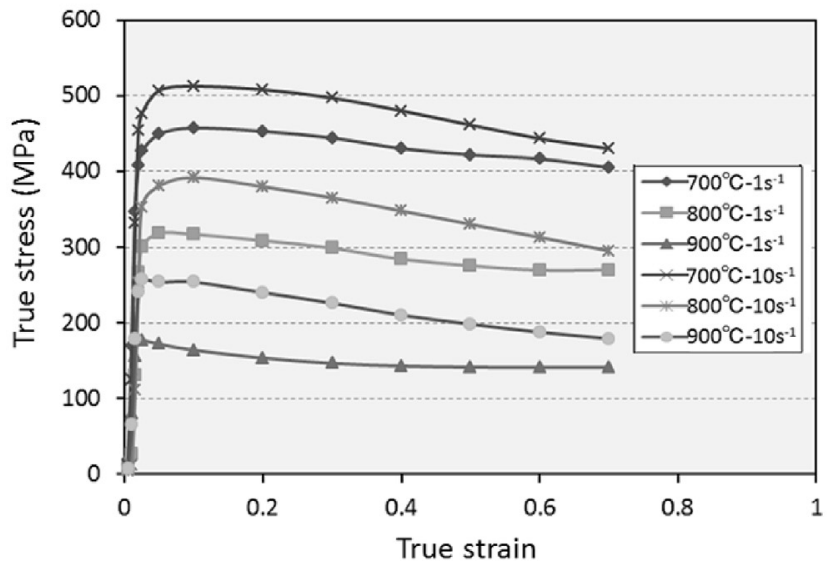

Fig. 2. Corrected stress-strain curves at testing temperatures of $700{ }^{\circ} \mathrm{C}, 800^{\circ} \mathrm{C}, 900{ }^{\circ} \mathrm{C}$ and strain rates of (a) $10^{-3} \mathrm{~s}^{-1}, 10^{-2} \mathrm{~s}^{-1}, 10^{-1} \mathrm{~s}^{-1}$ and (b) $1 \mathrm{~s}^{-1}, 10 \mathrm{~s}^{-1}$.

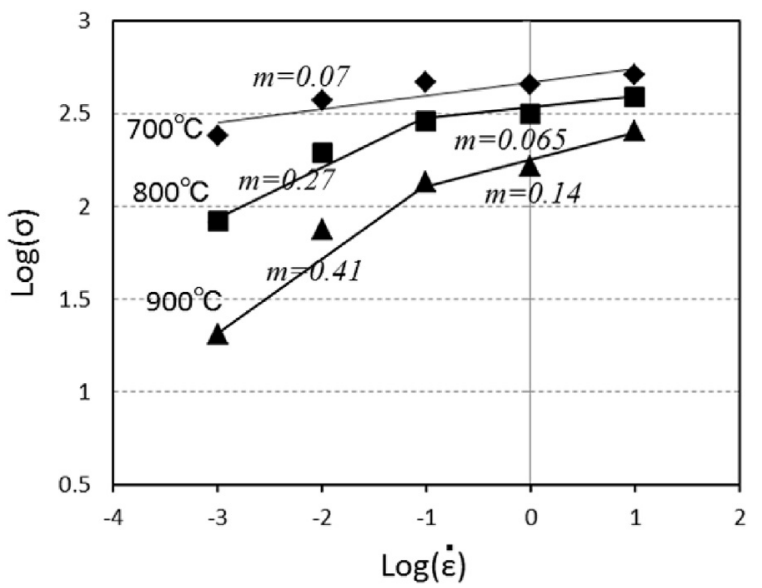

Fig. 3. Relationship between flow stress (at $\varepsilon$ of 0.1 ) and strain rate in the log-log scale at different testing temperatures.

$\dot{\varepsilon}=A \sigma^{n} \exp \left(-\frac{Q}{R T}\right)$

where $A$ is the material constant, $R$ is gas constant, and $n=(1 / m)$ is the stress exponent. The estimated $Q$ value (in the $(\alpha+\beta)$ region) in this work is $341.7 \mathrm{~kJ} / \mathrm{mol}$, which is similar to the $Q$ values previously reported in Ti-64 alloy [22], but much greater than the self- diffusion activation energy ( $153 \mathrm{~kJ} / \mathrm{mol}$ ) in $\beta$-titanium alloy [23]. The higher values of $Q$ in this work than the $Q$ (self-diffusion activation energy) are attributable to the occurrence of DRX of the $\alpha$ phase.

\subsubsection{Deformed microstructure after forging}

The deformed microstructures are summarized in Fig. 4. The microstructures of the $\alpha$ phase are given in the EBSD-grain boundary maps (black line: high angle boundary, green line: low angle boundary). In Fig. 4, the black phase corresponds to the $\beta$ phase. The microstructures having coarse equiaxed-grains are observed at the testing conditions of $800{ }^{\circ} \mathrm{C}-10^{-3} \mathrm{~s}^{-1}, 900{ }^{\circ} \mathrm{C}$ $10^{-3} \mathrm{~s}^{-1}$ and $900^{\circ} \mathrm{C}-10^{-2} \mathrm{~s}^{-1}$. According to the $m$ values, these testing conditions correspond to the region where superplasticity occurs. In other testing conditions, the microstructure consists of the deformed grains (in elongated morphology) and fine equiaxed DRX-grains, implying that the DRX is a dominant mode for microstructural conversion. Herein, it can be seen that the fraction of deformed grains (in the elongated morphology) tends to be higher with an increasing strain rate and decreasing temperature. Hereafter, the estimated DRX fractions would be applied to optimization of the material parameter in the JMAK formula (as indicated in section 4.1).

During deformation, a microstructure is recognized to have evolved due to the occurrence of DRX or CDRX [22,24]. Typical deformed microstructures during deformation at $700{ }^{\circ} \mathrm{C}-10 \mathrm{~s}^{-1}$ in this work are given in Fig. 5. It can be observed that the subgrain formation (surrounded by low angle boundary) is enhanced with an increasing strain. Furthermore, from Fig. 5(d), the straining is found to result in the formation of fine equiaxed grains with a high angle boundary. Therefore, as similar to the case with other Ti alloys, the DRX in the Ti-64 alloy with an equiaxed $(\alpha+\beta)$ starting microstructure is enhanced in a continuous manner and accompanied by subgrain formation and its subsequent rotation.

Hereafter, the Zener-Hollomon $(Z)$ parameter given by

$Z=\dot{\varepsilon} \exp \left(\frac{Q}{R T}\right)$

(where $R$ is the gas constant $(8.314 \mathrm{~J} /(\mathrm{mol} \mathrm{K})), T$ is the absolute temperature and $Q$ is the activation energy $(341.7 \mathrm{~kJ} / \mathrm{mol})$ for hot deformation in the $(\alpha+\beta)$ region), indicating the temperature compensated strain rate parameter is used for kinetic analyzing. Fig. 6 summarizes the relationship of DRX-grain size (for both low angle grain and high angle grain) and $Z$ parameter in Log-Log scale. The average grain sizes were determined by measuring the sizes of high-angle-boundary-grains and low-angle-boundary-grains, respectively. Linear relationships are clearly seen for both highand low-angle-grains, indicating that the grain size itself is dependent on the thermally activated process for controlling finegrains formation. Furthermore, there is the higher value in the slope for high angle grains in the lower $Z$ region (as shown in plots with empty circle) because the size of the DRX-high-angle-grain in this lower $Z$ region is supposed to be strongly affected by grain growth (under a static and dynamic-manner). From Fig. 6, the relationships between the DRX grain size and $Z$ parameter can be summarized as follows:

$d_{D R X(\text { high angle grain })}(\mu \mathrm{m})=125.49 Z^{-0.134}(\log (Z)<15.2)$

$d_{D R X(\text { high angle grain })}(\mu \mathrm{m})=1.847 Z^{-0.013}(\log (Z) \geq 15.2)$ 


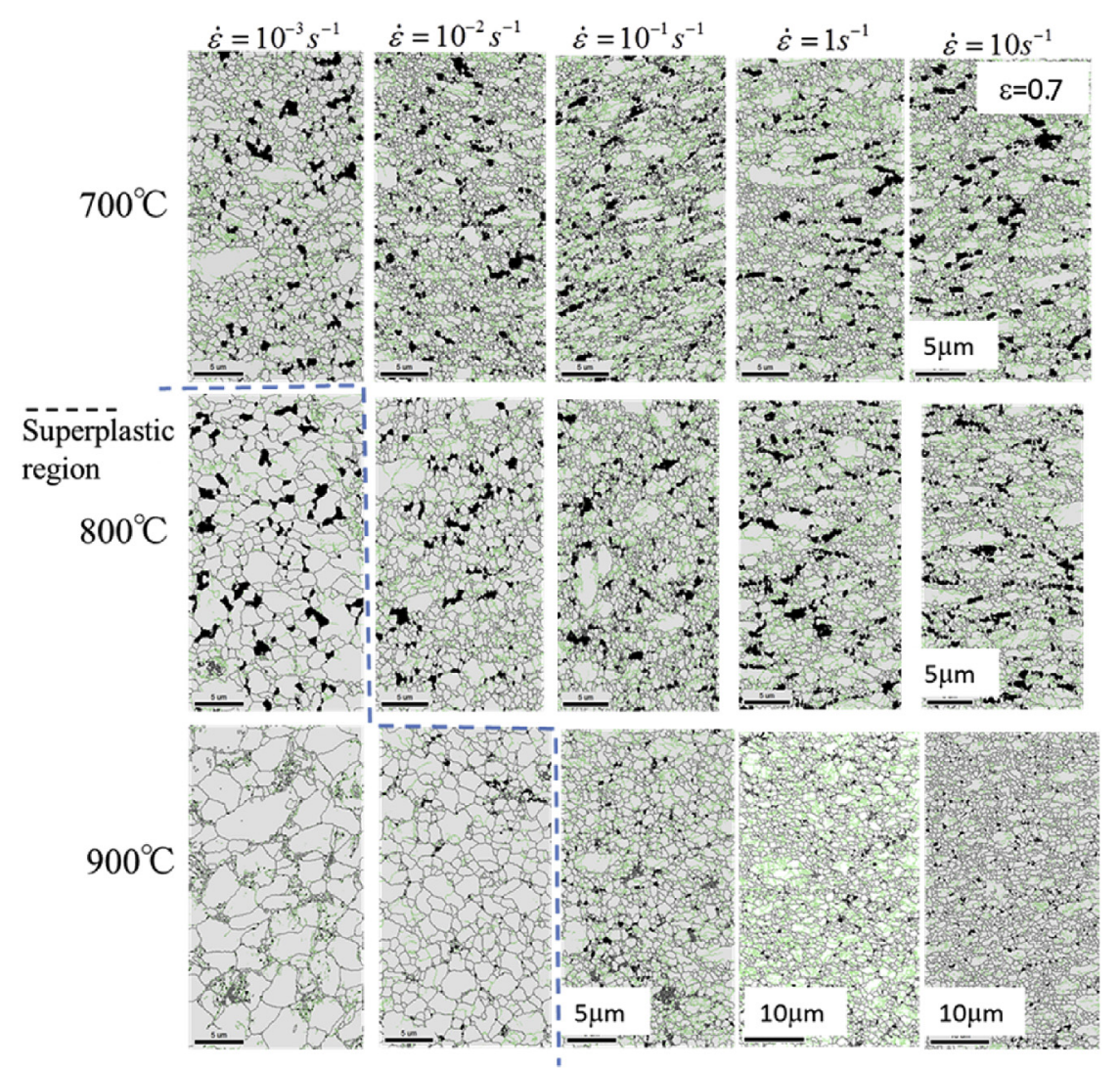

Fig. 4. Microstructures (i.e, EBSD-grain boundary images) at the central location of specimen after compression test (at a height true strain of 0.7 and at different testing conditions).

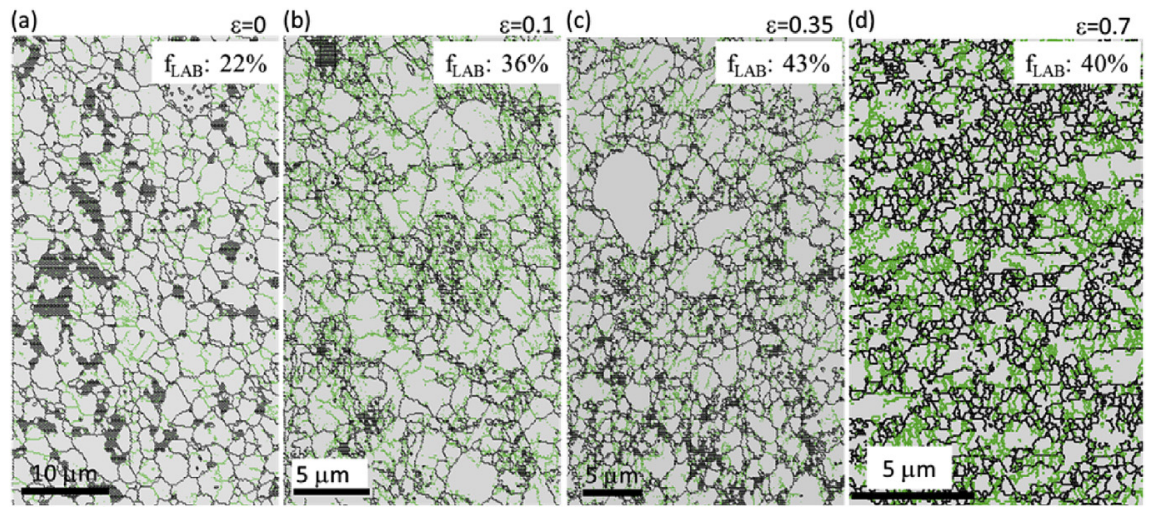

Fig. 5. EBSD-grain boundary images (at central location of specimen) of Ti-64 alloy compressed at $700{ }^{\circ} \mathrm{C}-10 \mathrm{~s}^{-1}$ and at a height based on the true strains of (a) 0 , (b) 0.1 , (c) 0.35 and (d) 0.7 .

$d_{D R X(\text { low angle grain })}(\mu \mathrm{m})=4.004 Z^{-0.061}$

\subsubsection{Processing map}

In order to characterize forging property, construction of the processing maps according to the dynamic materials model $[17,18]$ is available. This model considers that the work piece is a power dissipater and that the instant power dissipated for a given $\dot{\varepsilon}$ consists of two complementary parts: The $G$ content and the $J$ cocontent, which are related to the temperature rise and microstructural dissipation, respectively.
The efficiency of power dissipation $\eta$ with normalizing the instantaneous $J$ with the maximum value of $J_{\max }$ can be defined as:

$\eta=\frac{J}{J_{\max }}=\frac{2 m}{1+m}$

According to the criterion proposed by Ziegler [25], continuum criterion for the occurrence of flow instability $(\xi(\dot{\varepsilon}))$ is given by:

$\xi(\dot{\varepsilon})=\frac{\partial \ln [m /(1+m)]}{\partial \ln \dot{\varepsilon}}+m<0$

in which the equation considers the principle of the maximum rate of entropy production. Herein, flow instability occurs when $\xi(\dot{\varepsilon})$ 


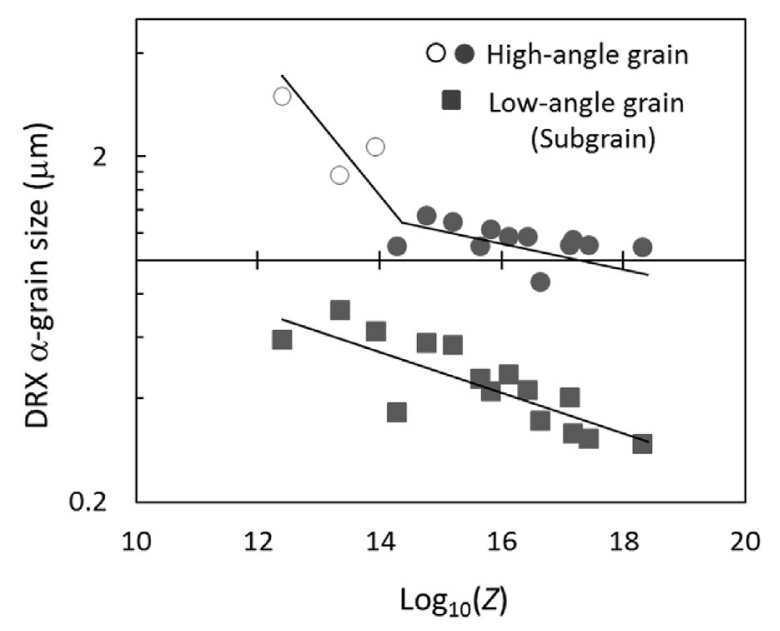

Fig. 6. Relationship between Zener-Hollomon ( $Z$ ) parameter and the DRX $\alpha$-grain size for high angle grain and low angle grain. The sizes of high- and low-angle grains are expressed by circle plots and square plots, respectively. The filled circle corresponds to the DRX-grain-size and the hollow circle does the grain size affected by not only the DRX behavior but also the dynamic grain growth behavior.

becomes negative while a value ranging from 0 to 1 for $\xi(\dot{\varepsilon})$ is judged to be metastable manner. The processing maps were identified by variation of $\eta$ and $\xi(\dot{\varepsilon})$ with deformation temperature and strain rate.

Fig. 7 summarizes the processing maps (at a true strain of 0.6 ) of the Ti-64 alloy with the equiaxed $(\alpha+\beta)$ starting microstructure. Shaded areas represent the metastable domains $(-1<\xi(\dot{\varepsilon})<0)$ or the unstable domains $(\xi(\dot{\varepsilon})<-1)$. The obtained processing map characteristic is indeed similar to the case in the previous report on Ti-64 alloy [26,27]. The optimum power dissipation efficiency for Ti alloys can be estimated to be in the range of $40-50 \%$ based on the correlation of optimum power dissipation efficiency in the dynamic recrystallization domain with normalized stacking fault energy (SFE) for various metals [17].

According to this consideration with the instability map, the optimum processing window can be identified in the region at strain rates less than $10^{-2} \mathrm{~s}^{-1}$ for all testing temperatures in which

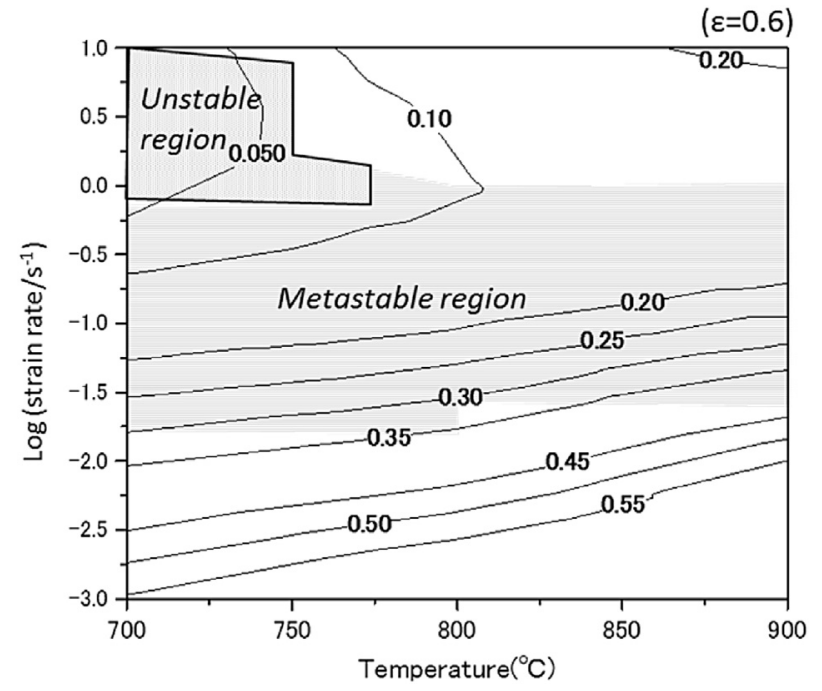

Fig. 7. Processing map for the Ti-6Al-4V alloy with the equiaxed $(\alpha+\beta)$ starting microstructure, at a height true strain of 0.6 . Shaded areas represent the metastable- or the unstable domains. the process window corresponds to superplastic deformation region. The relatively higher $\eta$ values exhibiting stable- or metastable-tendencies are also given at strain rates ranging from $10^{-1} \mathrm{~s}^{-1}$ to $10^{-2} \mathrm{~s}^{-1}$. In fact, this process window corresponds to the region where DRX (dominated by continuous manner) frequently occurs. In contrast, the region for temperatures below $750{ }^{\circ} \mathrm{C}$ and at higher strain rate corresponds to the domain with the lower $\eta$ and unstable region, indicating that adiabatic shear banding deformation is dominant for this condition. Indeed, the localized deformation zone with shear band formation is confirmed in the deformed specimen tested at $700{ }^{\circ} \mathrm{C}-10 \mathrm{~s}^{-1}$. At the higher strain rate region in Fig. 7, it can also be noted that the plastic deformation proceeds from unstable manner to stable manner as the testing temperature increases. This implies that the adiabatic effect decreases with increasing testing temperature, even though the lower power dissipation efficiency remains at the higher strain rate region.

\section{Predictions of microstructures and forging properties by FEM analysis combined with various constitutive laws}

\subsection{Microstructure predicted by FEM analysis combined with various constitutive laws}

In this study, the characteristic of DRX behavior is described by Johnson-Mehl-Avrami-Kolmogorov (JMAK) equation in the following [28]:

$X_{D R X}=1-\exp \left[-\beta_{d} \times\left(\frac{\varepsilon-\varepsilon_{C}}{\varepsilon_{0.5}}\right)^{k_{d}}\right]\left(\varepsilon \geq \varepsilon_{C}\right)$

where $X_{D R X}$ is the DRX fraction, $\beta_{d}$ and $k_{d}$ are material constants, $\varepsilon$ is the true strain, and $\varepsilon_{\mathrm{c}}$ is the critical strain for DRX initiation. The $\varepsilon_{0.5}$ is calculated as:

$\varepsilon_{0.5}=a_{1} d_{0}^{n 1} \dot{\varepsilon}^{m 1} \exp \left(\frac{Q_{1}}{R T}\right)$

$\beta_{D}=\beta_{0} \exp \left(-\frac{Q_{2}}{R T}\right)$

where $\dot{\varepsilon}$ is the strain rate, and $a_{1}, n_{1}, m_{1}, k_{d}, Q_{1}, Q_{2}$, and $\beta_{0}$ are material constants. The term $d_{0}(\mu \mathrm{m})$ is the initial grain size before deformation. The critical strain $\left(\varepsilon_{\mathrm{c}}\right)$ is determined by the peak strain $\left(\varepsilon_{\mathrm{p}}\right)$ (which is experimentally identified) as follows:

$\varepsilon_{\mathrm{C}}=0.8 \times \varepsilon_{\mathrm{p}}$

And the critical strain $\left(\varepsilon_{c}\right)$ is summarized by the relationship with the $Z$ parameter as follows:

$\varepsilon_{C}=C_{1} Z^{n 2}$

The material constants in the above equations are determined on the basis of the minimization of the sum of the errors between the experimental- and calculated data. The obtained material constants are given in Table 1.

With respect to the modeling of the grain growth, the static grain growth behavior is expressed in the following equation of Eq.

Table 1

Material constants for the constitutive models of dynamic recrystallization.

\begin{tabular}{lllllllll}
\hline$a_{1}$ & $n_{1}$ & $m_{1}$ & $Q_{1}(\mathrm{~J} \mathrm{~mol})$ & $k_{d}$ & $\beta_{0}$ & $Q_{2}(\mathrm{~J} \mathrm{~mol})$ & $C_{1}$ & $n_{2}$ \\
\hline 0.354 & 0.0779 & 0.0361 & 15973 & 2 & 1.071 & 7008 & 0.0269 & 0.0191 \\
\hline
\end{tabular}


Table 2

Material constants for the constitutive models of grain growth.

\begin{tabular}{llll}
\hline$k_{0}$ & $\mathrm{Q}_{g b g}(\mathrm{~J} \mathrm{~mol})$ & $C_{2}$ & $n_{3}$ \\
\hline $2.62 \times 10^{7}$ & 220000 & 0.0093 & 0.233 \\
\hline
\end{tabular}

(12) according to the Lifshitz-Slyosov-Wagner (LSW) theory [29]. The term $d_{\alpha}$ is grain size $(\mu \mathrm{m})$ of $\alpha$ ophase. The $n$ value (in the following) is set to 3 because of the deformation condition in this work is in the $(\alpha+\beta)$ region.

$d_{\alpha}^{n}-d_{\alpha 0}^{n}=d_{\alpha}^{3}-d_{\alpha 0}^{3}=K_{d}\left(t-t_{0}\right)$

in which the subscript ' 0 ' indicates the quantities at the initial stage and $K_{d}$ denotes the coarsening rate constant. The term $K_{d}$ is defined as the temperature dependent material constant and expressed as following Arrhenius relationship:

$K_{d}=K_{0} \exp \left(-\frac{Q_{g b g}}{R T}\right)$

in which $K_{0}$ and $Q_{g b g}$ are the material constants.

It is well recognized that the grain growth rate in dynamic conditions (under plastic deformation) is higher than that in static conditions, due to the strain (or stress) induced effects. Therefore, the dynamic grain growth rate of $K_{d}{ }^{\prime}$ is defined by the multiplication with the weighting factor of $n^{\prime}$. The term $n^{\prime}$ is expressed as following relationship with $Z$ parameter:

$n^{\prime}=C_{2} Z^{n_{3}}$

and

$K_{d}^{\prime}=n^{\prime}\left[K_{0} \exp \left(-\frac{Q_{g b g}}{R T}\right)\right]$

Summarizing the above equations, the grain size of the $\alpha$ phase $\left(d_{\alpha \text { (growth) }}\right)$ which considers the effect of grain growth can be summarized as following relationship:

$$
\begin{aligned}
\left(d_{\alpha(\text { growth })}\right)^{3}= & {\left[K_{0} \exp \left(-\frac{Q_{g b g}}{R T}\right)\right] t+n^{\prime}\left[K_{0} \exp \left(-\frac{Q_{g b g}}{R T}\right)\right] t } \\
& +d_{0}^{3}
\end{aligned}
$$

In order to identify the material constants for modeling the grain growth, the grain growth data was referred according to Ref. [30] for static growth and Refs. [31,32] for dynamic growth, followed by optimization of the material constants by non-linear regression analysis. Additionally, the determined material constants are given in Table 2.

Fig. 8 summarizes the relationship between the DRX fraction and the true strain for the experimental result, when tested at $700{ }^{\circ} \mathrm{C}$ (shown by symbols) and the predicted result (shown by solid line) according to Eq. (8). The results obtained are in a good agreement with the experimental results. The change in the DRX fraction is observed to be dependent on the strain, temperature and strain rate. Additionally, the DRX kinetic curves are characterized by an S-shape.

The microstructural evolution model (as stated above) has been implemented into the finite-element software of DEFORM-3D (ver. 10.2) through a user-subroutine followed by simulation of isothermal compression. Fig. 9 shows distributions of (a) effective strain, (b) the predicted DRX ratio and (c) the predicted average grain size $\left(d_{\text {ave }}\right)$ of the $\alpha$ phase for compression at $900{ }^{\circ} \mathrm{C}-1 \mathrm{~s}^{-1}$ and at the true strain height of 0.7 . Herein, the $d_{\text {ave }}$ is represented by:

$d_{a v e}=X_{D R X} d_{D R X}+\left(1-X_{D R X}\right) d_{0}$

where $d_{0}$ is initial grain size of $\alpha$ phase of $2.25 \mu \mathrm{m}$ with consideration of the grain growth in terms of Eq. (17).

As shown in Fig. 9(a), there is a heterogeneous distribution that strains are locally accumulated especially at central region. Additionally, the less strain region, called the dead-zone, is seen in Region $B$. Herein, the effective strain in Region $A$ reaches the value of 1.5 , and there is a decrease from Region A to the Region C. Although the distributions of effective strain rate and temperature are not given in Fig. 9, heterogeneous distributions have been also confirmed. With respect to the distribution of the DRX ratio (Fig. 9(b)), DRX is found to have frequently occurred $70 \%-80 \%$ in Region A, whereas no occurrence of DRX is in the dead-zone of the Region B. The distribution of the DRX ratio is found to exhibit good correlation with that of the effective strain (Fig. 9(a)). Moreover, with respect to the grain size distribution (Fig. 9(c)), in correlation with the result of the DRX ratio, the finer grain size is seen in Region A where the DRX frequently occurs, while there are coarser size distributions in Regions B and C.

For comparison with the experimental result, microstructures in Regions A, B and C (expressed in Fig. 9), as observed by SEM, are given in Fig. 10. The DRX accompanied by the fine-grain-formation is seen to be frequently activated in Region A (Fig. 10(a)). On the other hand, equiaxed grain-microstructure having the similar grain size for the initial conditions is observed in Region B (Fig. 10(b)). In addition, the microstructure in Region $C$ exhibits coarser grained microstructure than that in Region A. This reveals that the experimental result is in good agreement with the simulated result (Fig. 9). Thus, it can be found that the constitutive models in this work allow to accurately predict the microstructural evolution.

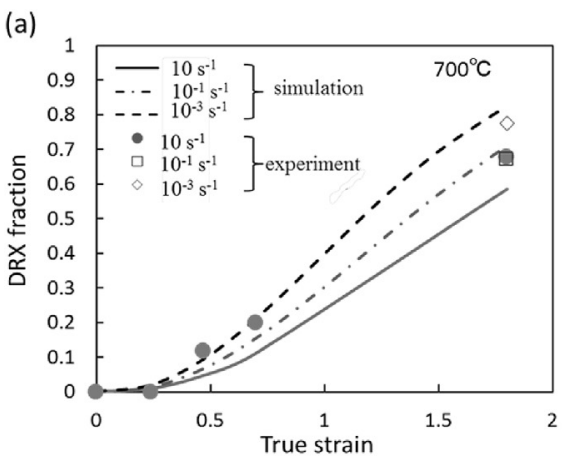

(b)

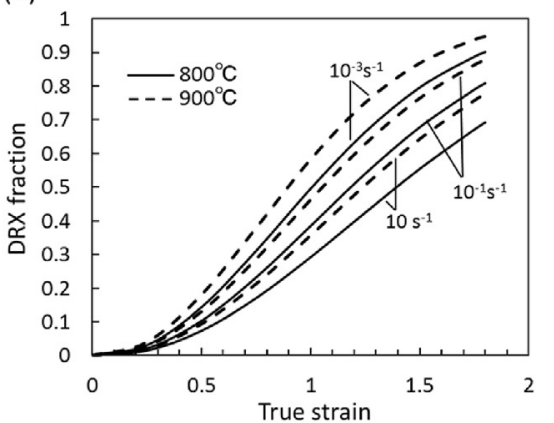

Fig. 8. Predicted and experimental fraction of the DRX as a function of true strain. Testing temperatures of (a) $700{ }^{\circ} \mathrm{C}$ and (b) $800{ }^{\circ} \mathrm{C}, 900{ }^{\circ} \mathrm{C}$. 

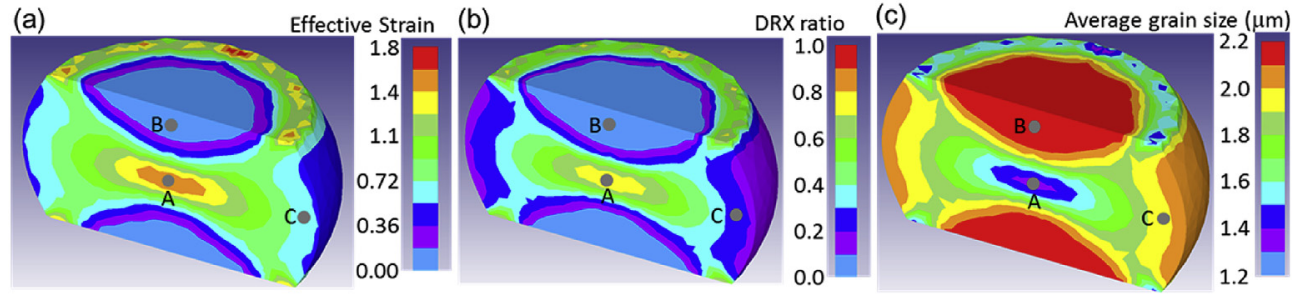

Fig. 9. Distribution from the FEM analysis for (a) Effective strain, (b) DRX fraction and (c) Average grain size for the $\alpha$ phase at compression of $900{ }^{\circ} \mathrm{C}-1 \mathrm{~s}^{-1}$ and a height for the true strain of 0.7 .

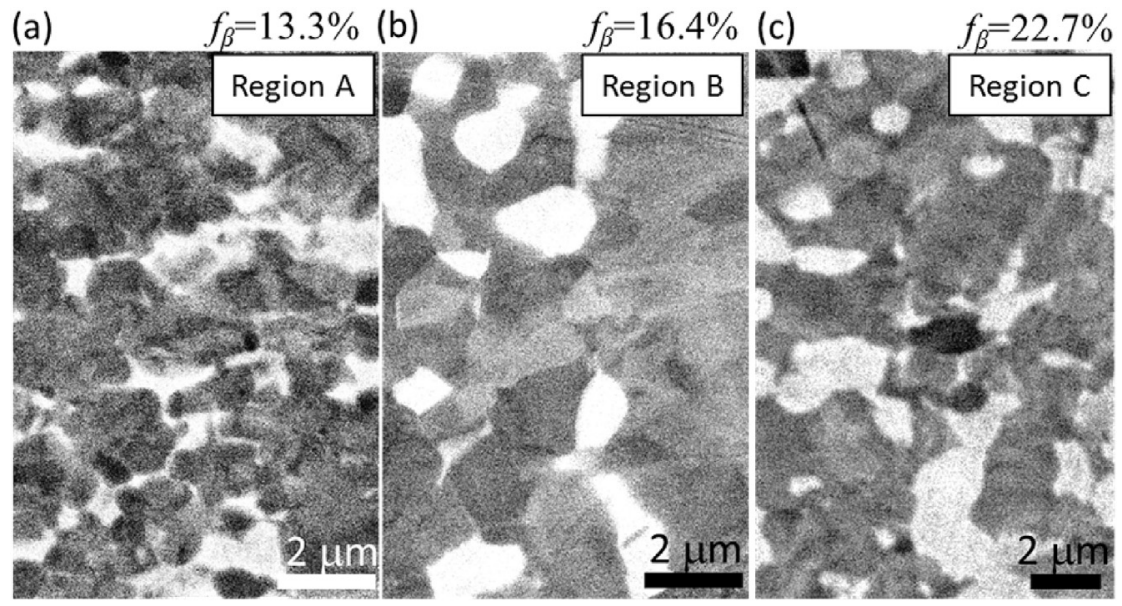

Fig. 10. SEM micrographs of Ti-6Al-4V alloy after deformation of $900{ }^{\circ} \mathrm{C}-1 \mathrm{~s}^{-1}$ and a height for the true strain of 0.7 : (a) Region A, (b) Region B and (c) Region C in Fig. 9.

\subsection{Prediction of processing map characteristic}

As abovementioned in Section 3.1.3, the efficiency of power dissipation is expressed in Eq. (6) with parameter of the $m$ value. Hence, $m$ is an important variable for predicting the efficiency of power dissipation. The experimentally determined $m$ values (at true strains of 0.1 and 0.6 ) are summarized as a function of the $Z$ parameter (in log scale), as shown in Fig. 11. There are no apparent changes in $m$ with the change in true strain. Therefore, we can judge that $m$ is not influenced by the change in strain. The splinefitting curve by approximation (in a cubic polynomial) is also indicated in Fig. 11, and the formula of this approximation is as follows:

$$
\begin{aligned}
m= & -0.0044\left(\log _{10}(Z)\right)^{3}+0.2235\left(\log _{10}(Z)\right)^{2}-3.824 \log _{10}(Z) \\
& +22.169
\end{aligned}
$$

In order to predict distributions of the processing map characteristics, the constitutive equations of (6), (7) and (18) were implemented in the DEFORM system. For predicting the processing map characteristics, the $m$ value in the simulation is set to be constant value of 0.5 if the calculated $m$ value obtained by Eq. (18) is above 0.5. Fig. 12(a)(b) illustrates the distributions of (a) the efficiency of power dissipation, as calculated by Eq. (6), and (b) the instability criteria, as evaluated by Eq. (7), which is forged for the conditions at $900{ }^{\circ} \mathrm{C}-1 \mathrm{~s}^{-1}$. With respect to the instability criteria (Fig. 12(b)), plastic deformation is found to occur in a metastable manner in the vicinity of the central region, whereas, the stable manner is given in the dead-zone region. This is due to the correlation with the distribution of effective strain rate that the extremely lower effective strain rate exhibits in the vicinity of the dead-zone. When associated with the distribution of the effective strain rate, it results in an apparent higher efficiency of power dissipation in the vicinity of the dead-zone (edge of specimen) region rather than that at the central region. However, this result does not correlate with the distribution of the microstructures of the DRX fraction and grain size (as shown in Fig. 9(b)(c)) since Eq. (6) does not consider the effect of straining. Therefore, modification of Eq. (6) is required in order to simulate the more reliable $\eta$, which also considers the effect of straining. The modified efficiency of

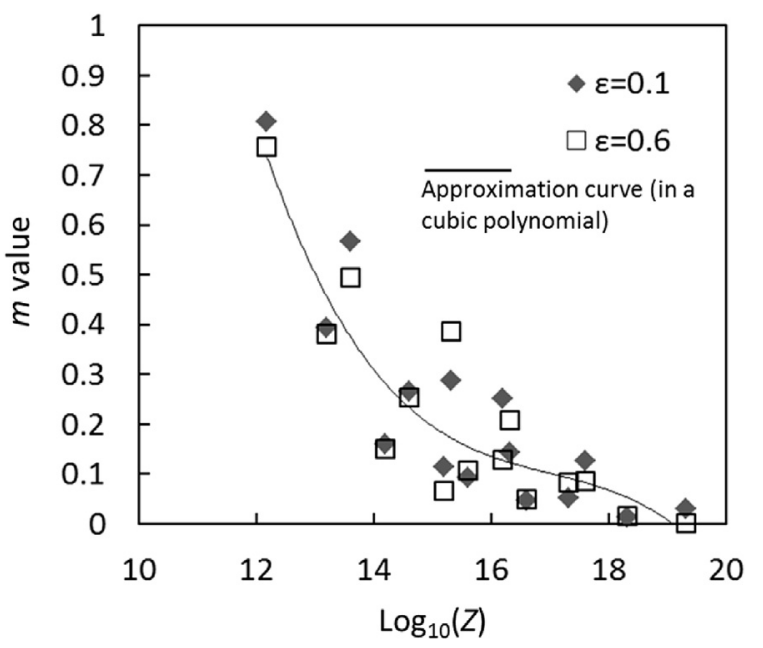

Fig. 11. Relationship between the strain rate sensitivity $(m)$ and the Zener-Hollomon $(Z)$ parameter (in log scale) for the true strains of 0.1 and 0.6 based on an approximation curve. 
(a)

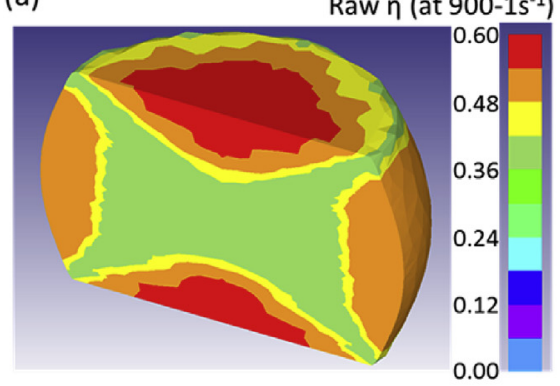

(c)
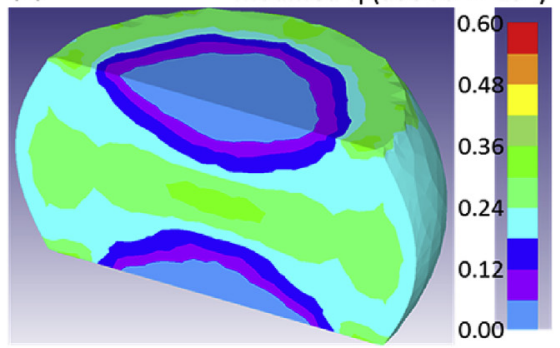

(b)

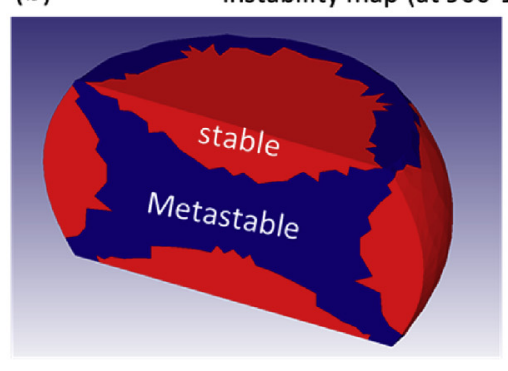

(d)

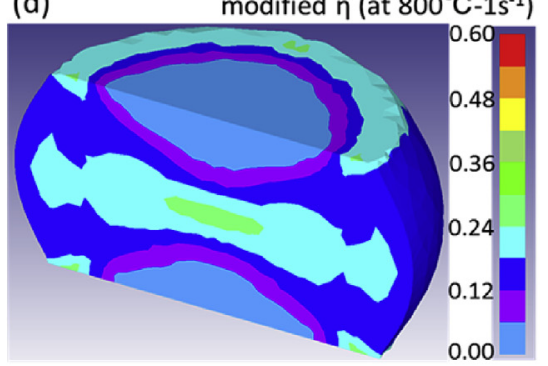

Fig. 12. Distributions from the FEM analysis for (a) Efficiency of power dissipation, (b) Instability map, and (c), (d) Modified efficiency of power dissipation at testing conditions of (a)(b)(c) $900{ }^{\circ} \mathrm{C}-1 \mathrm{~s}^{-1}$ and (d) $800{ }^{\circ} \mathrm{C}-1 \mathrm{~s}^{-1}$.

power dissipation of $\eta^{\prime}$ is herein defined as follows:

$\eta^{\prime}=\eta \times f(\varepsilon)$

$f(\varepsilon)=1-\exp (-\varepsilon)$

where $\varepsilon$ is effective strain.

In this equation, the efficiency of the power dissipation is modified by multiplying with the term $f(\varepsilon)$ which is a similar type of equation as the JMAK equation. Thus, the efficiency of the power dissipation can contain the effect of the strain through the $f(\varepsilon)$ parameter. Fig. 12(c) and (d) show the distributions of the modified efficiency of the power dissipation of $\eta^{\prime}$ deformed at $900{ }^{\circ} \mathrm{C}-1 \mathrm{~s}^{-1}$ and $800^{\circ} \mathrm{C}-1 \mathrm{~s}^{-1}$. Despite the difference when compared with the distribution of $\eta$ (in Fig. 12(a)), the good correlation can be seen between $\eta^{\prime}$ and the occurrence of dynamic recrystallization (Fig. 9(b)). Therefore, in order to express the more reliable processing map behavior, it seems reasonable to adopt the multiplication of $\eta$ by the term $f(\varepsilon)$.

\subsection{Room-temperature-strength (or hardness) after forging}

During hot deformation, plastic deformation leads to the accumulation of dislocations, while DRV annihilates dislocations. In this study, the Kocks-Mecking equation (K-M model) [33] was employed to describe the dislocation evolution. In the K-M model, the dislocation accumulation rate $(d \rho / d \varepsilon)$ due to plastic deformation is proportional to $\rho^{1 / 2}$, and the dislocation annihilation due to thermal-activated-process of DRV is proportional to $\rho$. The equation of $\mathrm{K}-\mathrm{M}$ model is as follows:

$\frac{d \rho}{d \varepsilon}=k_{1} \sqrt{\rho}-k_{2} \rho$

where the first and the second term represent the dislocation accumulation by work hardening and dislocation annihilation via DRX, respectively. The terms $k_{1}$ and $k_{2}$ are the rates of dislocation storage and dislocation annihilation via DRX, respectively. DRV is a thermally activated process induced by dislocation-slip or climb; hence, the parameter $k_{2}$ in Eq. (21) is a function of the temperature and strain rate and is expressed as follows:

$k_{2}=k_{0} \dot{\varepsilon}^{m_{2}-1} \exp \left(-\frac{Q_{C L}}{R T}\right)$

where the $Q_{C L}$ is the activation energy for dislocation cross-slip and recombination.

The macroscopic flow stress $(\sigma)$ during isothermal hot compression is related to the average dislocation density, which can be expressed by:

$\sigma=0.5 \mu(T) b \sqrt{\rho}$

The parameter $b$ is the burgers vector of the $\alpha$ phase (= $\left.2.95 \times 10^{-10} \mathrm{~m}\right)$ and the parameter $\mu(T)$ is the temperature dependent shear modulus (GPa) [12];

$\mu(T)=49.02-\frac{5.821}{e^{181 / T}-1}$

The material constants were optimized on the basis of the corrected experimental stress-strain curves by non-linear regression analysis. Herein, the material parameters were determined by assuming the parameter $\rho_{0}$ (initial dislocation density) to be $1.0 \times 10^{10} \mathrm{~m}^{-2}$. The determined material parameters of $k_{1}, k_{2}, m_{2}$, and $Q_{C L}$ are $3.37 \times 10^{11}, 6.33 \times 10^{5}, 0.937$, and $39182(\mathrm{~J} / \mathrm{mol})$ respectively. The distribution of the dislocation density $(\rho)$ estimated by the above $\mathrm{K}-\mathrm{M}$ model in specimen compressed at $900{ }^{\circ} \mathrm{C}-$ $1 \mathrm{~s}^{-1}$ and at a height strain of 0.7 is shown in Fig. 13(a). Similar to the distributions of the effective strain, the DRX ratio, and the average grain size (from Fig. 9) shown in Fig. 13(a) exhibits heterogeneous distribution where the dislocation accumulates especially within the central region. The higher dislocation density results in a higher stored energy, and leads to an enhancement of the DRX. Hence, the good correlation between the distributions of the dislocation density ( $\rho$ ) (Fig. 13(a)) and the DRX ratio (Fig. 9(b)) is revealed.

As abovementioned, the typical microstructural evolutions of the DRX, the grain size and the dislocation density were 
(a)

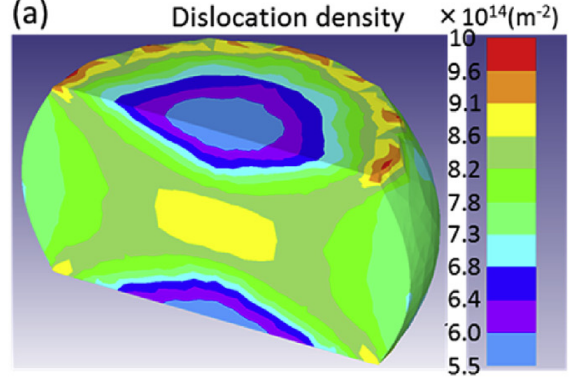

(b)

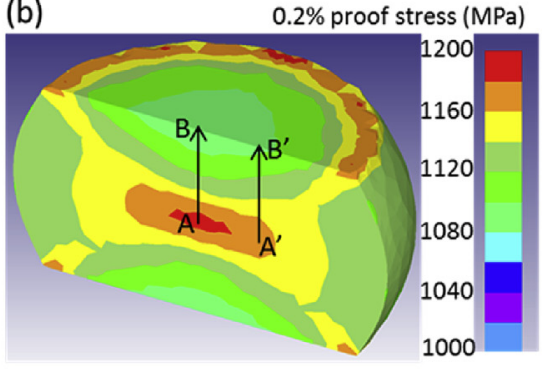

Fig. 13. Distributions from the FEM analysis of (a) Dislocation density and (b) $0.2 \%$ proof stress at room temperature after forging. Compression condition is $900{ }^{\circ} \mathrm{C}-1 \mathrm{~s}^{-1}$ and a height of the true strain of 0.7 .

successfully predicted. Hereafter, by considering the abovepredicted microstructural data, we work to establish the model for predicting the room temperature strength of the product after forging. In estimation of the yield stress $(0.2 \%$ proof stress) of the forged product, the classical models of the Hall-Petch relation and the Bailey-Hirsch relation were applied to the estimation of strength. Regarding the effect of grain size on strength, the increment of yield stress $\left(\sigma_{H-P}\right)$ according to Hall-Petch effect is expressed as:

$\sigma_{H-P}=K_{y} d_{\alpha}^{-0.5}$

where $K_{y}$ and $d_{\alpha}$ are the Hall-Petch coefficient and $\alpha$-grains size, respectively. According to previous reports revealing the Hall-Petch effect of Ti-64 alloy [34-36], the Hall-Petch coefficient ranges from $0.135 \mathrm{MPa} /\left(\mathrm{m}^{-1 / 2}\right)$ to $0.46 \mathrm{MPa} /\left(\mathrm{m}^{-1 / 2}\right)$. This work estimates the Hall-Petch relation with the coefficient of $0.25 \mathrm{MPa} /\left(\mathrm{m}^{-1 / 2}\right)$. The dislocation contribution to strengthening $\left(\sigma_{B-H}\right)$, according to the Bailey-Hirsch relation, is stated as follows:

$\sigma_{B-H}=0.5 \mu b \rho^{1 / 2}$

It can be summarized the microstructural effect on $0.2 \%$ proof stress $\left(\sigma_{0.2}\right)$ as follows:

$\sigma_{0.2}=\sigma_{0}+\sigma_{H-P}+\sigma_{B-H}$

where the $\sigma_{0}$ is the friction stress ( $=760 \mathrm{MPa}$ [36]). The estimated $0.2 \%$ proof stress after forging at $900{ }^{\circ} \mathrm{C}-1 \mathrm{~s}^{-1}$ and at a height strain of 0.7 is given in Fig. 13(b), revealing the heterogeneous distribution of strength. The highest proof stress of approximately $1180 \mathrm{MPa}$ is exhibited within the central region where the DRX is frequently activated, while the lower proof stress around $1080 \mathrm{MPa}$ is seen within the dead-zone region (in upper- and downward-region in specimen). In order to compare this simulated result with the experimental strength in the forged specimen, the experimental hardness results obtained by Vickers-hardness testing were converted to a yield stress according to Hill's law [37]. The simulated $0.2 \%$ proof stress and the experimental yield stress (converted from the Vickers hardness) at the locations along $A-B$ and $A^{\prime}-B^{\prime}$, as illustrated in Fig. 13(b), are summarized in Fig. 14. There is a difference of approximately $60 \mathrm{MPa}-70 \mathrm{MPa}$ between the experimental result and the simulated result. The effect of the $\beta$-fraction on the change in strength is not considered in Eq. (27). As seen in Fig. 10, the $\beta$-fraction is indeed different depending on location in a compressed specimen, which is attributable to the difference in temperature during deformation and the cooling rate after forging. According to the relationship between the $0.2 \%$ proof stress and the $\beta$-fraction [38], the effect of the $\beta$-fraction on the strength $\left(\sigma_{M-R}\right)$ can be expressed as following equation:

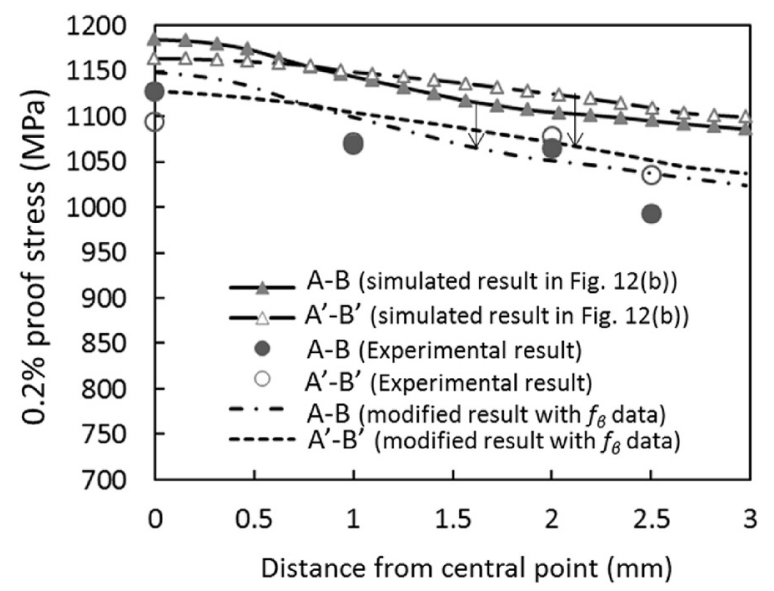

Fig. 14. Experimental and simulated $0.2 \%$ proof stress along the $A$ to $B$ line and $A^{\prime}$ to $B^{\prime}$ line shown in Fig. 13.

$\sigma_{M-R}=940.7-272.3 f_{\beta}$

where the $f_{\beta}$ is $\beta$-fraction, and the right term of $940.7 \mathrm{MPa}$ corresponds to the $0.2 \%$ proof stress of $\alpha$ phase. In Fig. 13 , the results after this modification into Eq. (27) are also given. From Fig. 13, we can confirm that the modification with the effect of the $\beta$ phase results in close approximations to the experimental values. Moreover, a similar tendency regarding the change in the proof stress along $\mathrm{A}-\mathrm{B}$ and $\mathrm{A}^{\prime}-\mathrm{B}^{\prime}$ lines is observed for the experimental and the simulated results. Hence, the room-temperature strength after forging is found to be reasonably simulated by the association with the classical models of the Hall-Petch relation, the Bailey-Hirsch relation, and the simple mixture rule.

As abovementioned, the constitutive models embedded into the DEFORM-3D platform were found to provide an approximate agreement with the experimental results (e.g., microstructures, processing map characteristic, and room temperature strength after forging). For additional, highly accurate prediction of the microstructure, a further direction of this study will be to focus on the appropriate combination of phenomenological models and internal-variable-based constitutive models.

\section{Summary}

The present work focused on a series of predictions regarding microstructures, DRX behavior, the change in grain size, and dislocation density as well as the processing map characteristic during forging of the Ti-64 alloy at temperatures in the $(\alpha+\beta)$ region, which is in combination with finite-element-method (FEM) 
analysis. The obtained results are as follows.

(1) Experimental study of the forging of the Ti-64 alloy at the $(\alpha+\beta)$ region reveals that superplastic behavior is dominant for a condition of lower strain rate and higher temperature, whereas DRX under continuous method is dominant for the relatively higher strain-rate, lower temperature region. The relationship between the DRX grain size and $Z$ parameter exhibits a linear-relationship, indicating that the grain size itself is dependent on the thermally activated process for controlling fine-grains formation. The processing map obtained in this work is in good agreement with the behavior of the microstructural conversion. That is, a higher efficiency of power dissipation $(\eta)$ is given in the region where DRX frequently occurs.

(2) Constitutive models for microstructural prediction, processing map characteristics and room temperature strength were established through an optimization process (based on experimental results) of the material parameters. The DRX behavior could be reasonably expressed in terms of the JMAK constitutive equation. The modified equation for efficiency of the power dissipation enables the simulation to more reliably correlate with the DRX behavior. Thus, the simulated results according to these constitutive models are in good agreement with the experimental results. Furthermore, it is found that the strength at room temperature after forging can be reasonably simulated by classical constitutive models according to the Hall-Petch relation, the Bailey-Hirsch relation, and simple mixture rule. Additionally, this simulated strength also exhibits satisfactory similarities with the experimental results. Thus, a series of constitutive models, as established in this work, is found to enable the reliable prediction of microstructures and properties of Ti-6Al-4V alloy after forging in the $(\alpha+\beta)$ region.

\section{References}

[1] R.D. Doherty, D.A. Hughes, F.J. Humphreys, J.J. Jonas, D. Juul Jensen, M.E. Kassner, W.E. King, T.R. McNelley, H.J. McQueen, A.D. Rollett, Current issues in recrystallization: a review, Mater. Sci. Eng. A238 (1997) 219-274.

[2] F.J. Humphreys, M. Hatherly, Recrystallization and Related Annealing Phenomena, Pergamon Press, Oxford, 1995.

[3] C.M. Sellars, Modelling microstructural development during hot rolling, Mater. Sci. Technol. 6 (1990) 1072-1081.

[4] H.J. McQueen, Development of dynamic recrystallization theory, Mater. Sci. Eng. A 387-389 (2004) 203-208.

[5] A. Laasraoui, J.J. Jonas, Recrystallization of austenite after deformation at high temperature and strain rates-analysis and modeling, Metall. Trans. A 22 (1991) 151-160.

[6] S.I. Kim, Y. Lee, D.L. Lee, Y.C. Yoo, Modelling of AGS and recrystallisation fraction of microalloyed medium carbon steel during hot deformation, Mater. Sci. Eng. A 355 (2003) 384-393.

[7] H.S. Jeong, J.R. Cho, H.C. Park, Microstructure prediction of Nimonic 80A for large exhaust valve during hot closed die forging, J. Mater. Proc. Tech. $162-163$ (2005) 504-511.

[8] F. Chen, Z. Cui, J. Chen, Prediction of microstructural evolution during hot forging, Manuf. Rev. 1 (6) (2014) 1-21.

[9] R. Ding, Z.X. Guo, Microstructural evolution of a Ti-6Al-4V alloy during $\beta$ phase processing: experimental and simulative investigations, Mater. Sci. Eng. 365A (2004) 172-179.

[10] C. Wu, H. Yang, H.W. Li, Simulated and experimental investigation on discontinuous dynamic recrystallization of a near- $\alpha$ TA15 titanium alloy during isothermal hot compression in $\beta$ single-phase field, Trans. Nonferrous Met. Soc. China 24 (2014) 1819-1829.
[11] Z.C. Sun, H. Yang, G.J. Han, X.G. Fan, A numerical model based on internalstate-variable method for the microstructure evolution during hot-working process of TA15 titanium alloy, Mater. Sci. Eng. A 527 (2010) 3464-3471.

[12] X.G. Fan, H. Yang, Internal-state-variable based self-consistent constitutive modeling for hot working of two-phase titanium alloys coupling microstructure evolution, Int. J. Plast. 27 (2011) 1833-1852.

[13] E. Alabort, D. Putman, R.C. Reed, Superplasticity in Ti-6Al-4V: characterisation, modelling and applications, Acta Mater. 95 (2015) 428-442.

[14] G.Z. Quan, G.C. Luo, J.T. Liang, D.S. Wu, A. Mao, Q. Liu, Modelling for the dynamic recrystallization evolution of $\mathrm{Ti}-6 \mathrm{Al}-4 \mathrm{~V}$ alloy in two-phase temperature range and a wide strain rate range, Comput. Mater. Sci. 97 (2015) 136-147.

[15] D.L. OuYang, M.W. Fu, S.Q. Lu, Study on the dynamic recrystallization behavior of Ti-alloy $\mathrm{Ti}-10 \mathrm{~V}-2 \mathrm{Fe}-3 \mathrm{Al}$ in $\beta$ processing via experiment and simulation, Mater. Sci. Eng. A619 (2014) 26-34.

[16] K.L. Wang, M.W. Fu, S.Q. Lu, X. Li, Study of the dynamic recrystallization of Ti-6.5 Al-3.5 Mo-1.5Zr-0.3Si alloy in $\beta$-forging process via finite element method modeling and microstructure characterization, Mater. Des. 32 (2011) $1283-1291$.

[17] Y. Prasad, T. Seshacharyulu, Modeling of hot deformation for microstructural control, Int. Mater. Rev. 43 (1998) 243-258.

[18] Y. Prasad, Modeling of dynamic material behavior in hot deformation: forging of Ti-6242, Metall. Mater. Trans. 15A (1984) 1883-1892.

[19] Y. Li, E. Onodera, A. Chiba, Friction coefficient in hot compression of cylindrical sample, Metall. Mater. Trans. 51A (2010) 1210-1215.

[20] Y. Li, E. Onodera, H. Matsumoto, Y. Koizumi, S. Yu, A. Chiba, Development of novel methods for compensation of stress-strain curves, ISIJ Int. 51 (2011) $782-787$.

[21] M. Mataya, V. Sackschewsky, Effect of internal heating during hot compression on the stress-strain behavior of alloy 304L, Metall. Mater. Trans. 25A (1994) 2727-2752.

[22] I. Weiss, S.L. Semiatin, Thermomechanical processing of alpha titanium alloys-an overview, Mater. Sci. Eng. A 263 (1999) 243-256.

[23] I. Weiss, S.L. Semiatin, Thermomechanical processing of beta titanium alloys-an overview, Mater. Sci. Eng. A243 (1998) 46-65.

[24] S. Zherebtsov, M. Murzinova, G. Salishchev, S.L. Semiatin, Spheroidization of the lamellar microstructure in Ti-6Al-4V alloy during warm deformation and annealing, Acta Mater. 59 (2011) 4138-4150.

[25] H. Ziegler, in: I.N. Sneddon, R. Hill (Eds.), Progress in Solid Mechanics, vol. 4, 1965, pp. 91-193.

[26] T. Seshacharyulu, S.C. Medeiros, W.G. Frazier, Y.V.R.K. Prasad, Hot working of commercial Ti-6Al-4V with an equiaxed $\alpha-\beta$ microstructure: materials modeling considerations, Mater. Sci. Eng. A284 (2000) 184-194.

[27] J. Luo, P. Ye, M.Q. Li, L.Y. Liu, Effect of the alpha grain size on the deformation behavior during isothermal compression of Ti-6Al-4V alloy, Mater. Des. 88 (2015) 32-40.

[28] C.M. Sellars, J.A. Whiteman, Recrystallization and grain growth in hot rolling, Metal. Sci. 13 (1979) 187-194.

[29] J.W. Martin, R.D. Doherty, B. Cantor, Stability of Microstructure in Metallic System, Cambridge University Press, Cambridge, United Kingdom, 1997.

[30] C.H. Johnson, S.K. Richter, C.H. Hamilton, J.J. Hoyt, Static grain growth in a microduplex Ti-6Al-4V alloy, Acta Mater. 47 (1999) 23-29.

[31] C.A. Sargent, A.P. Zane, P.M. Fagin, A.K. Ghosh, S.L. Semiatin, Low-temperature coarsening and plastic flow behavior of an alpha/beta titanium billet material with an ultrafine microstructure, Metall. Mater. Trans. 39A (2008) 2949-2964.

[32] S.L. Semiatin, P.N. Fagin, J.F. Betten, A.P. Zane, A.K. Ghosh, G.A. Sargent, Plastic flow and microstructure evolution during low-temperature superplasticity of ultrafine Ti-6Al-4V sheet material, Metall. Mater. Trans. 41A (2010) 499-512.

[33] U.F. Kocks, H. Mecking, Physics and phenomenology of strain hardening: the FCC case, Prog. Mater. Sci. 48 (3) (2003) 171-273.

[34] R. Armstrong, I. Codd, R.M. Douthwaite, N.J. Petch, The plastic deformation of polycrystalline aggregates, Phil. Mag. 7 (1962) 45-58.

[35] Y. Long, H. Zhang, T. Wang, X. Huang, Y. Li, J. Wu, H. Chen, High-strength Ti-6Al-4V with ultrafine-grained structure fabricated by high energy ball milling and spark plasma sintering, Mater. Sci. Eng. A 585 (2013) 408-414.

[36] J. Thomas, M. Groeber, S. Ghosh, Image-based crystal plasticity FE framework for microstructure dependent properties of Ti-6Al-4V alloys, Mater. Sci. Eng. A 553 (2012) 164-175.

[37] R. Hill, The Mathematical Theory of Plasticity, Oxford University Press, 1967.

[38] Y. Sun, W. Zeng, Y. Han, Y. Zhao, G. Wang, M.S. Dargusch, P. Guo, Modeling the correlation between microstructure and the properties of the Ti-6Al-4V alloy based on an artificial neural network, Mater. Sci. Eng. A 528 (2011) $8757-8764$. 